\title{
Stratum Intermedium
}

National Cancer Institute

\section{Source}

National Cancer Institute. Stratum Intermedium. NCI Thesaurus. Code C33628.

A layer of condensed cells in the developing tooth that is located between the inner enamel epithelium and the stellate reticulum. 